\title{
Familial hypercholesterolemia with early coronary atherosclerotic heart disease: A case report
}

\author{
YANHONG LUO ${ }^{1}$, MIN ZHENG $^{2}$, YUTIN ZHANG ${ }^{2}$, SHUANG HE $^{2}$, LEI ZHANG $^{2}$, \\ HUICHAO SUN ${ }^{2}$, XIAOYAN LIU ${ }^{2}$, TAO TAN $^{3}$, HUA ZHU $^{3}$ and JIANFENG HE ${ }^{2}$ \\ Departments of ${ }^{1}$ Endocrinology and Genetic Metabolism and ${ }^{2}$ Cardiology, Children's Hospital Chongqing Medical University, \\ Chongqing 400014, P.R. China; ${ }^{3}$ Department of Surgery, Davis Heart and Lung Research Institute, \\ The Ohio State University Wexner Medical Center, Columbus, OH 43210, USA
}

Received April 2,2018; Accepted October 5, 2018

DOI: $10.3892 / \mathrm{etm} .2019 .7653$

\begin{abstract}
Patients with familial hypercholesterolemia usually present with high levels of serum low-density lipoprotein, xanthomas and early coronary artery disease. A 13 years old female patient was admitted to Children's Hospital of Chongqing Medical University presenting symptoms of heart failure. Laboratory tests showed that her cholesterol and low-density lipid levels were extremely high. Electrocardiogram test revealed that she had sinus tachycardia, QT lengthening and ST-T change. Multiple cardiac function abnormalities were diagnosed by echocardiogram. Multiple coronary artery stenosis was determined by computed tomography angiography. After the combination of lipid lowering, anti-thrombosis, and cardiac remodeling therapies, the patient's symptoms were significantly improved and the patient was discharged.
\end{abstract}

\section{Introduction}

Familial hypercholesterolaemia $(\mathrm{FH})$ is a rare genetic disorder, which affects around $0.2 \%$ of the population (1). The disease manifestations are very high levels of serum low-density lipoprotein (LDL), xanthomas and early coronary artery disease (CAD). The genetic mutations of lipid handling genes in patients lead to hyperlipidemia that usually develop into severe atherosclerosis and cause ischemia heart diseases and heart failure. Due to technical challenging and resource limitations, genetic screening is not possible to diagnose patients with $\mathrm{FH}$, especially in developing countries. Thus, establishment of an easy and low cost diagnostic approach for FH is needed.

Correspondence to: Dr Jianfeng He, Department of Cardiology, Children's Hospital Chongqing Medical University, 136 Zhongshan Er Road, Chongqing 400014, P.R. China

E-mail: 1534568197@qq.com

Key words: familial hypercholesterolemia, low-density lipoprotein, coronary artery disease, diagnosis
In the present study, we reported a FH patient admitted in our hospital with symptoms of heart failure. With physical and laboratory tests, the patient showed typical FH symptoms. Cardiac dysfunctions and coronary artery stenosis were further determined by electrocardiogram, echocardiogram and CT angiography. The patient was immediately treated with combination of lipid lowering, anti-thrombosis and cardiac remodel improving therapies. The patient was discharged when the heart failure symptoms were successfully controlled.

\section{Case report}

A 13 years old female patient was admitted to Children's Hospital of Chongqing Medical University (Chongqing, China) due to symptoms of heart failure, including lower limb swelling for 9 days, coughing and panic for 6 days. Upon arriving hospital, the patient felt heart palpitations, breath shortness, and fatigue after short walk. The lower extremity of the patient had pitting edema, which had been aggravated with oliguria and sweating for 9 days. In the course of the disease, there was a paroxysmal cough accompanied with the yellow mucus phlegm and bloody phlegm. None of the following symptoms was presented: wheezing and dyspnea, eyelid edema, gross hematuria, foam urine, chest tightness, chest pain, nausea, vomiting, abdominal distension, diarrhea, dizziness, headache, syncope, or fever. Based on her disease history, dermal yellow nodules were found right after her birth, they developed into nodular xanthoma during her growth.

Upon admitted in our hospital, physical examination was performed: Her body temperature was $36.6^{\circ} \mathrm{C}$, breath was $30 \mathrm{BPM}$, heart rate was $142 \mathrm{BPM}$, and blood pressures were 110/58 mmHg. The skin of double knees, left elbow, ankle, wrist, toe and coccygeal joint had hemispherical yellow eminence subcutaneous nodules (Fig. 1). The size of nodules ranged from $1.0 \times 2.0$ to $5.0 \times 6.0 \mathrm{~cm}$. The boundary was clear, texture was tough, and the surface was free from swelling and ulceration. The apex beat was located at the 5th intercostal space about $3.0 \mathrm{~cm}$ from left midclavicular line. No presentation of lifting type pulsation and pericardial friction was detected. The heart beat sounded slightly dull and arrhythmia could be detected. 

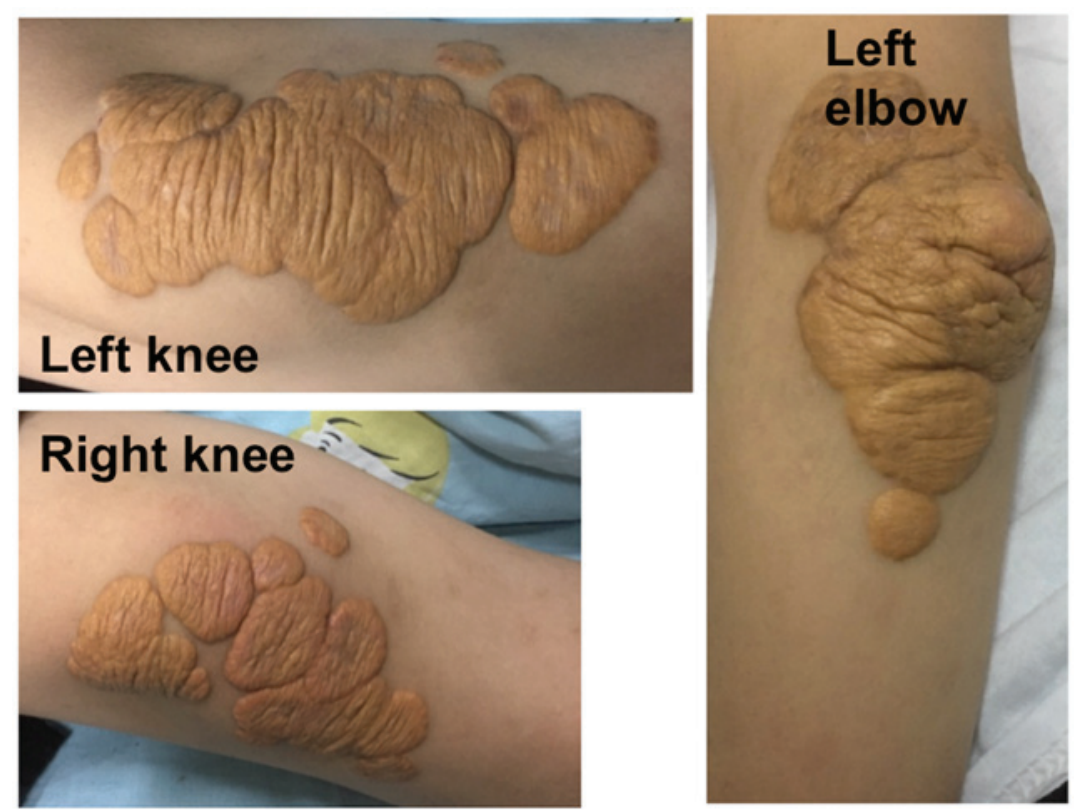

Figure 1. Photograph shows that skin xanthomas presented on skin of knees and left elbow.

Laboratory tests were performed: blood lipids were extremely high [total cholesterol (TC) $14.73 \mathrm{mmol} / 1$, high-density lipoprotein (HDL) $0.59 \mathrm{mmol} / 1$, LDL $13.44 \mathrm{mmol} / \mathrm{l}$, B type natriuretic peptide was $800 \mathrm{pg} / \mathrm{ml}$, lactic acid was $4.04 \mathrm{mmol} / 1$, blood amine was $61.7 \mathrm{~mol} / \mathrm{l}$, CRP was $59 \mathrm{mg} / \mathrm{l}$, PTC was $1.138 \mathrm{ng} / \mathrm{ml}$. In addition, the following tests were performed and showed normal: Blood routine, urine routine, fecal routine, liver and kidney function, electrolytes, myocardial markers, ESR, immunization, autoantibodies, blood glucose and blood gas tandem mass spectrometry.

Because the patients showed symptoms of heart failure, cardiac related tests were performed. First, electrocardiogram of the patient showed sinus tachycardia, QT lengthening, ST-T change (Fig. 2). Dynamic electrocardiogram test revealed that ventricular premature beat presented 2 times $/ 24 \mathrm{~h}$, atrial premature beat presented onceper $24 \mathrm{~h}$, the average heart rate was 97 beats per minute, and the heart rate variability was poor. Furthermore, cardiac morphology and function were determined by echocardiography (Fig. 3). Multiple pathological changes have been observed, including enlarged left ventricle, enlargement of left and right atrium, and right ventricle, decrease of ventricular septum and left ventricular posterior wall motion, and aortic sinus tube junction stenosis with hyperechoic structure with moderate regurgitation, moderate to severe mitral valve regurgitation, mild to moderate tricuspid valve regurgitation, decreased left ventricular systolic function, slightly widened inferior vena cava. In addition, abdominal B-ultrasound test revealed a slight swelling of the liver. In order to examine the cause of ischemic heart disease, chest CT angiography was performed (Fig. 4): There were multiple points of stenosis in the ascending aorta, left and right coronary artery and descending aorta, while CT of double lungs showed normal. The bifurcation of the left coronary artery was stenosis, the degree of stenosis was $93 \%$, the left anterior descending coronary artery had stenosis after diagonal branches, the degree of stenosis was $68 \%$, the upper right coronary artery (from the start $27.9 \mathrm{~mm}$ ) also had stenosis, the degree of stenosis was $42 \%$.
Except for the patients, no skin xanthoma and premature coronary heart disease were found in the family.

Based on the patient's medical history, clinical manifestation, blood lipid and cardiac test results. Diagnosis was made: the patient showed typical FH with coronary atherosclerotic heart disease. She had grad II cardiac insufficiency, nodular xanthoma, atherosclerosis, and vavular calcification. In order to confirm our diagnosis, a genetic test was prescribed to the patient. Unfortunately, the parents of the patient refused to perform the genetic test due to the financial difficulties.

Based on guidance from the 2014 European Atherosclerotic Association and the 2016 British HoFH management, the following treatments were prescribed to the patient: Sodium phosphocreatine $1.0 \mathrm{~g} \mathrm{IV}$, qd, 11 days, VitC $3.0 \mathrm{~g} \mathrm{IV}$, qd, 11 days, combined coenzyme needle 1 branches IV, qd, 11 days to nourish the myocardium. Milrinone $50 \mu \mathrm{g} / \mathrm{kg}$, slow intravenous injection for $10 \mathrm{~min}$ later, $0.5 \mu \mathrm{g} / \mathrm{kg} / \mathrm{min}, 6$ days, and maintain dobutamine $5 \mu \mathrm{g} / \mathrm{kg} / \mathrm{min}$ IV continued $2 \mathrm{~h}$, q8 h, 5 days, digoxin $0.125 \mathrm{mg} /$ time, po, qd for 6 days were used to enhance myocardial contractility. Oral Atorvastatin $20 \mathrm{mg} / \mathrm{time}, \mathrm{qN}$ for 6 days and diet control were used to lower blood lipid. Ezetimibe Tablets: $10 \mathrm{mg} / \mathrm{time}$, po, bid for 6 days were administrated to stablize plaque. Benazepril $10 \mathrm{mg} / \mathrm{time}$, po, qd for 5 days and metoprolol $6.25 \mathrm{mg} / \mathrm{time}$, po, bid for 2 days were used to improve myocardial remodeling. Spironolactone $20 \mathrm{mg} / \mathrm{time}$, po, bid for 11 days, furosemide $20 \mathrm{mg} /$ time, po, bid for 10 days and hydrochlorothiazide $25 \mathrm{mg} / \mathrm{time}$, po, bid for 1 day, diuresis. Aspirin $100 \mathrm{mg} / \mathrm{time}$, po, qd for 3 days and clopidogrel $50 \mathrm{mg}$, po, qd for 3 days were used to prevent thrombosis formation. After treatment for 11 days, the symptoms of the patient were improved, she was discharged.

After returning home, her symptom has been improved. The drugs were subscripted to her after discharge: Oral drug spironolactone $10 \mathrm{mg} /$ time qd for one year, hydrochlorothiazide $12.5 \mathrm{mg} / \mathrm{times}$, qd for one year, metoprolol $6.25 \mathrm{mg} / \mathrm{time}$, bid for one year, hydrogen chloropidogrel $25 \mathrm{mg} / \mathrm{time}$, qd 


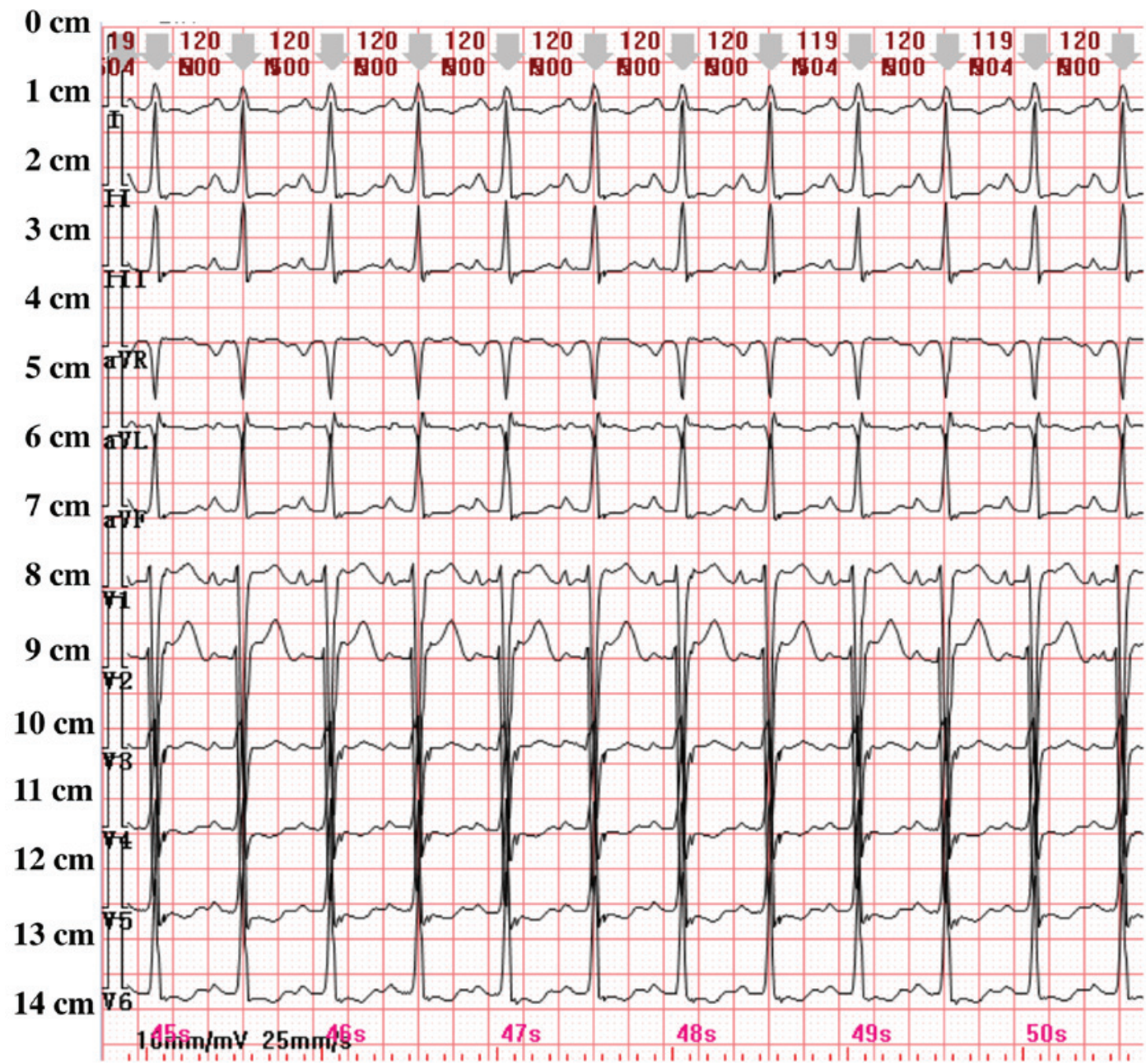

Figure 2. Electrocardiogram shows sinus tachycardia, QT lengthening, ST-T change.
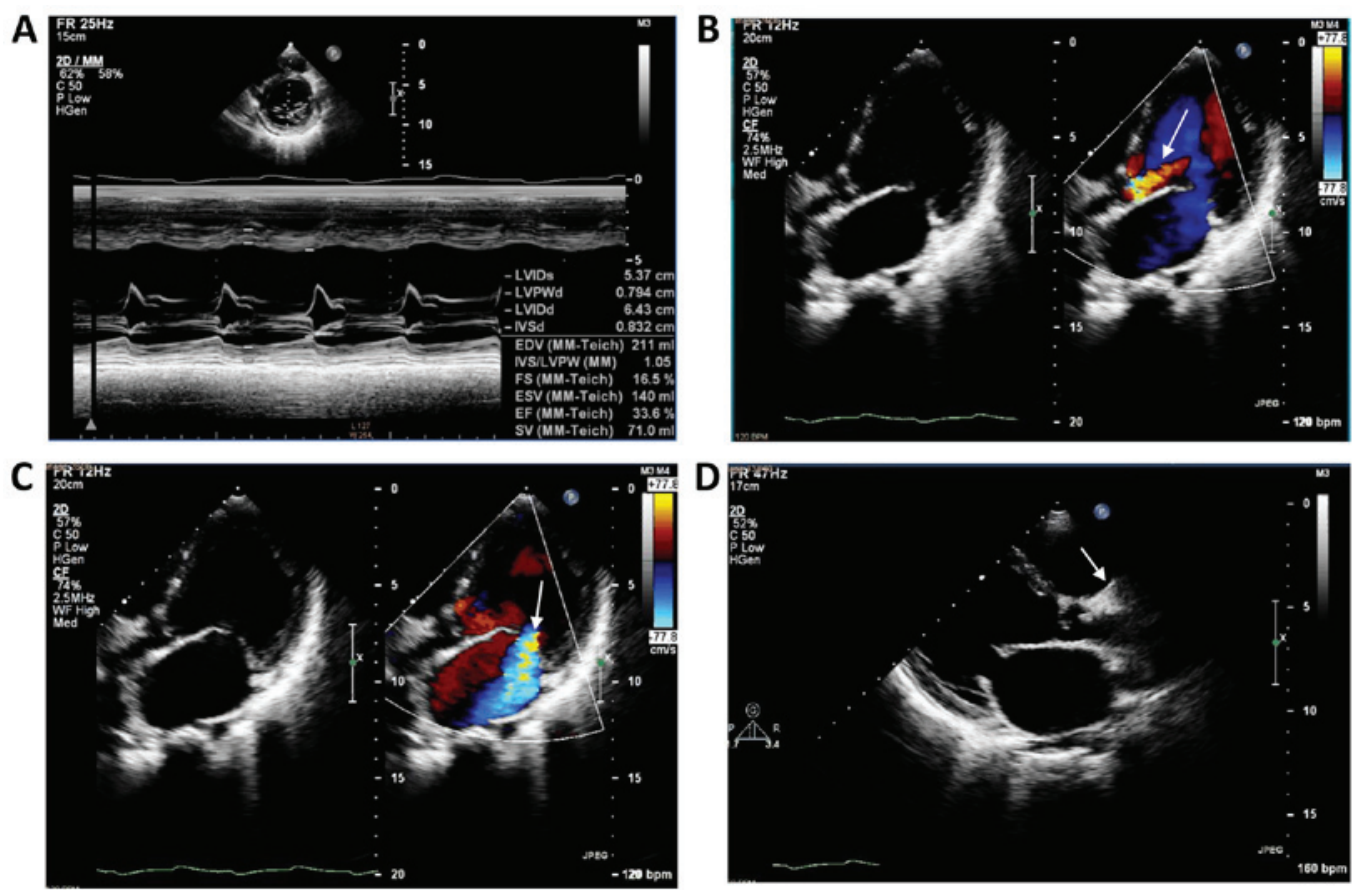

Figure 3. Echocardiogram pictures show reduced cardiac functions of the patient. (A) The left ventricle was significantly increased and the left ventricular systolic function decreased (EF 33.6\%, FS 16.5\%). (B) Left ventricular enlargement, left atrial enlargement and moderate reflux of aortic valve (white arrow). (C) The left ventricle was obviously enlarged, the left atrium increased, and the mitral regurgitation was moderate and severe (the white arrow indicates the mitral regurgitation). (D) Stenosis of the junction of the aortic sinus with hyperechoic structure (white arrow). 
A

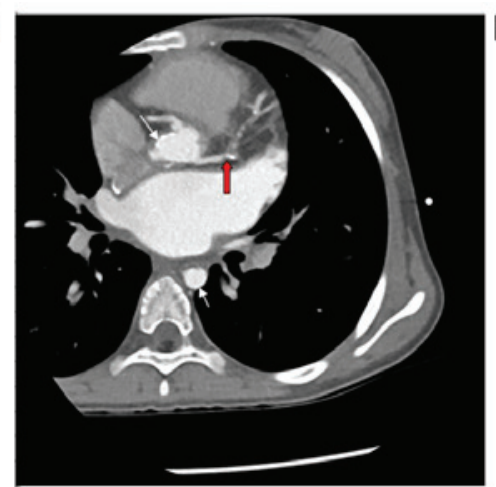

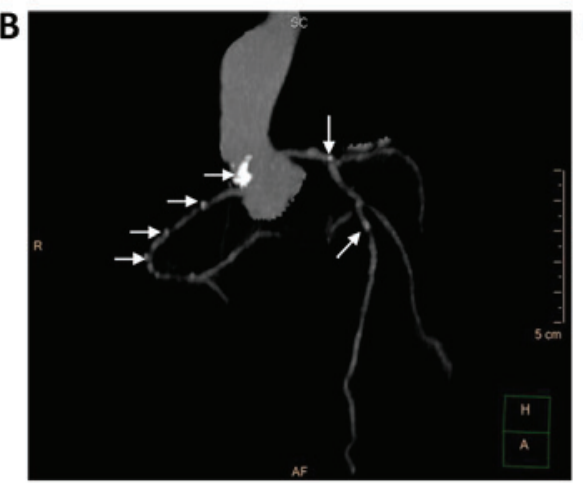

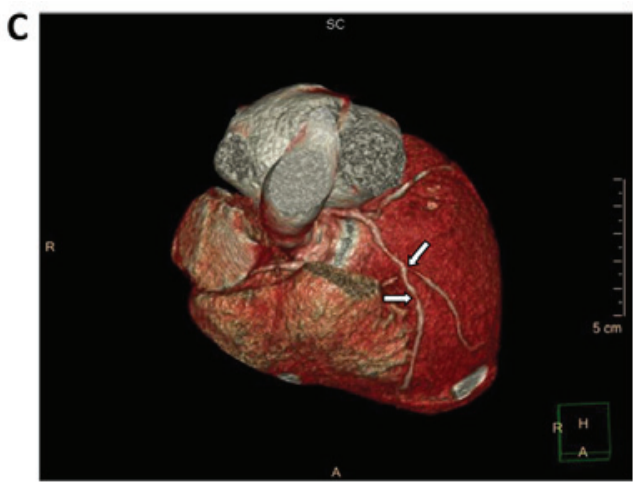

Figure 4. CT angiography shows multiple stenosis of aorta and coronary artery of the patient. (A) The ascending aorta, left and right coronary artery and descending aorta showed multiple point density and soft tissue density (white arrow), the bifurcation of the left coronary artery stenoses (red arrow). (B) The ascending aorta and left and right coronary artery showed multiple point density, as indicated by the white arrows. (C) Obvious stenosis of the anterior descending branch of the left coronary artery after the diagonal branch is separated (white arrow).

for one year, atorvastatin $20 \mathrm{mg} /$ time, $\mathrm{qN}$ for one year. After 1 year of treatment, the patient was retested for blood lipids (TC $10.03 \mathrm{mmol} / 1$, HDL $0.48 \mathrm{mmol} / 1$, LDL $852 \mathrm{mmol} / \mathrm{l}$ ), function of liver and kidney, electrolytes, blood glucose, blood coagulation, myocardial damage markers were normal, ECG was sinus tachycardia, and ST segment was low.

Ethical approval for the present study was obtained from Children's Hospital of Chongqing Medical University and oral informed consent was obtained from both the patient and the patient's parent.

\section{Discussion}

$\mathrm{FH}$ is an autosomal dominant genetic disease. The most common cause of $\mathrm{FH}$ is the mutations of the genes encoding lipid handling proteins, such as LDL receptor (LDLR), apolipoprotein B (ApoB) or proteins convertase subtilisin/Kexin/kexin9 (PCSK 9), resulting in LDL metabolism defects, and LDL cholesterol (LDL-C) levels are abnormally elevated in plasma. The FH patients usually have xanthoma in peripheral tissue, atherosclerosis and other clinical manifestations. Based on a FH study in Jiangsu, China, the Chinese FH diagnostic standard was established (2) (summarized in Table I). Based on this standard, the patient in current study met multiple criteria of $\mathrm{FH}$, including an early-onset coronary heart disease history and extremely high level of LDL-C (13.44 mmol/l), and xanthoma formation at the tendon sites. FH can further be divided into homozygote type $(\mathrm{HoFH})$ and heterozygote type (HeFH), where the $\mathrm{HoFH}$ is a serious rare disease (3). The prevalence of $\mathrm{HoFH}$ was $1 /(16-100) \times 10^{4}$, and the patients' serum LDL-C $>13 \mathrm{mmol} / \mathrm{l}$. If without any treatment, the HoFH patients usually die from coronary heart disease before the age of 30 (4). The incidence of coronary heart disease in patients with HoFH is 100 times higher than that of the normal population, and most of the patients with HoFH are able to develop in childhood and adolescence (5). The main clinical manifestations of HoFH are plasma LDL-C level is extremely high (approximately 6-8 times higher than normal), corneal arch, xanthoma at the tendon sites, premature coronary heart disease and aortic valve disease (6). Aortic valve disease is mainly caused by the involvement of the root of the aorta, which leads to the stenosis and calcification of the aorta on the upper part of the aortic valve and the aortic valve, and even the opening of the coronary artery (7-9). Except absent of corneal arch, the patients displayed all other symptoms.

The 2014 European Atherosclerotic Association (European Atherosclerosis Society, EAS) HoFH management guide proposed that the diagnosis of $\mathrm{HoFH}$ should be based on the standard of genetic diagnosis or in conformity with the clinical diagnostic criteria. The diagnostic genes include LDLR, ApoB, PCSK9, and LDLRAP1. The clinical diagnostic criteria are: LDL-C $>13 \mathrm{mmol} / \mathrm{l}$ before treatment or LDL-C $>8 \mathrm{mmol} / \mathrm{l}$ after treatment, and the presence of xanthoma in the tendon at the age of 10 or the level of parental LDL-C was consistent with heterozygote $\mathrm{FH}$ (HeFH) (3). The 2016 British HoFH management guide also recommends the use of genetic diagnostic or clinical diagnostic criteria, in which the genetic diagnostic criteria are basically the same as the EAS HoFH management guidelines. Children and adults diagnostic criteria are: Children LDL C>11 mmol/l and skin tendon xanthoma before 10 years of age; adult LDL-C $>13 \mathrm{mmol} / 1$ and there were obvious xanthoma of skin tendon, or LDL-C level reached clinical diagnosis standard, while parents were diagnosed as HeFH (8). Therefore, we diagnosed the patient in this study as $\mathrm{HoFH}$.

Finally, after literature search, we found one previous study that reported a 17 years old female patient. She had hypercholesterolemia on 2 year old, xanthoma at age of 9, and the clinical manifestations of coronary heart disease at age of 12 . In addition, her TC and LDL-C was significantly increased, the electrocardiogram has ST segment depression, cardiac color Doppler showed stenosis and mitral regurgitation. There was sign of heart failure. The aorta stenosis and coronary artery stenosis were detected by angiography. The genetic examination revealed that she has exon 541T $>C$ homozygous mutation of LDLR gene (9). Based on the clinical manifestation, we believe our results are in agreement with those reported by Zhao et al (9).

Thus, we showed the feasibility of a clinical diagnosis of $\mathrm{HoFH}$ without a genetic diagnosis. We believe this is extremely importantly in developing countries, where genetic diagnosis might be challenging and not available. In this case, we had successfully used lipid-lowering therapy, anti-thrombosis 
Table I. Chinese FH diagnostic criteria.

\begin{tabular}{lc}
\hline Standard & Score \\
\hline First degree relatives have a family history of early & 1 \\
onset coronary heart disease or vascular disease & \\
Individual history of early onset coronary heart & 2 \\
disease & \\
Personal history of early cerebrovascular disease & 1 \\
LDL-C, mmol/1 ${ }^{-1}$ & \\
$\geq 6.0$ & 8 \\
$5.0-5.9$ & 5 \\
$3.5-4.9$ & 3 \\
$2.5-3.4$ & 1 \\
FH diagnosis & \\
Diagnosis & $>8$ \\
Very likely & $6-8$ \\
Probably & $3-5$ \\
Impossible & $<3$ \\
\hline
\end{tabular}

FH, familial hyper cholesterolaemia; LDL-C, low-density lipoprotein-cholesterol.

therapy and cardiac remodeling therapy to improve cardiac function of the patient.

According to the clinical diagnostic criteria, only about $1 / 4$ of FH cases can be predicted. Most patients were diagnosed until middle age, thus missing the chance of early treatments. It has been estimated that there are about 26 million potential $\mathrm{FH}$ patients in China (10), but the rate of diagnosis and treatment in clinics is still very low (11). According to the international FH foundation, population screening should be based on age, sex and specific LDL-C level. Systematic screening strategy is very important for identifying $\mathrm{FH}$ proband, which is the key to discover new cases for family members (12). Therefore, we should perform blood lipids, electrocardiogram, echocardiography and carotid ultrasound examination on parents, siblings and other family members, and complete family genetic testing, in order to identify $\mathrm{FH}$ patients and perform early treatment.

The current treatment options for FH include lifestyle changes, drug therapy and liver transplantation. Lifestyle changes are the most important aspect of lipid-lowering therapy, including reducing uptake of saturated fatty acids and cholesterol, eating cellulose rich foods, stopping smoking and controlling salt consumption, proper physical activity, and weight loss. Conventional lipid-lowering drugs include statins and ezetimibe. The National Lipid Association of the United States suggested that the level of LDL-C in patients with FH decreased to below $2.5 \mathrm{mmol} / \mathrm{l}$ or decreased by $>50 \%$ compared with that before treatment (12). But HoFH is a serious hereditary disease. Even when multiple drugs combined with lifestyle intervention, it is still difficult to achieve LDL-C compliance (13). The EAS HoFH management guide recommends that $\mathrm{HoFH}$ patients take high-intensity tolerable dose statins as initial treatment, gradually combine with other types of lipid-lowering drugs such as ezembo, and PCSK9 inhibitor, or plasma lipoprotein replacement, in addition, some patients may choose to undergo liver transplantation (14). In recent years, with the in-depth study of the mechanism of cholesterol metabolism, new target drugs are emerging. The most representative new drugs are mainly two oligonucleotide inhibitors (mipomersen and lomitapide) (15) and two PCSK9 monoclonal antibody inhibitors (alirocumab and evolocumab) (16). Mipomersen and lomitapide can significantly reduce blood lipids in patients with $\mathrm{HoFH}$, but the cost is high and the incidence of adverse reactions is high. In contrast, PCSK9 inhibitors are better choices at present because of their therapeutic effects and high potency ratio.

Clearly, our case report has limitations. One of them was there was no genetic test for the patient to finally confirm the case was a HoFH at the gene level. Luckily, some clinical evidences strongly suggested it was a HoFH case. First, the immediate family members are all normal without known skin xanthoma, indicating they might be heterozygous carriers. Second, the symptoms of the patients are consistent to the diagnostic criteria from both the 2014 European Atherosclerotic Association and the 2016 British HoFH management guidance. Most importantly, after following treatment of $\mathrm{HoFH}$, the patient has recovered and was finally discharged. Thus, we feel our case is deserved to be achieved and valuable for the physicians from developing countries, where the genetic tests might not be feasible. The other limitations include that we do not have information for the family history of coronary heart disease neither did we check the patient's second-degree family members for the symptom. We failed to obtain this important information due to quick improvement of the patient's symptom and discharge of the patient.

\section{Acknowledgements}

Not applicable.

\section{Funding}

The present study was supported by internal funding from Chongqing Medical University.

\section{Availability of data and materials}

The datasets used and/or analyzed during the current study are available from the corresponding author on reasonable request.

\section{Authors' contributions}

YL, TT, HZ and JH participated in the manuscript writing; YL, TT, MZ, YZ, SH and LZ participated in data collection; XL participated in manuscript editing and data analysis; $\mathrm{HZ}$ and $\mathrm{JH}$ participated in the data analysis and manuscript revision. All authors have read and approved the manuscript.

\section{Ethics approval and consent to participate}

The study has been approved by IRB of Children's Hospital of Chongqing Medical University. 


\section{Patient consent for publication}

The patient provided oral consent for publication of this study.

\section{Competing interests}

The authors declare that they have no competing interests.

\section{References}

1. Selvan JP, Uthaman B, Abushaban L and Jebaraj R: Homozygous familial hypercholesterolemia with generalized arterial disease. Medical principles and practice: international journal of the Kuwait University. Health Sci Centre 16: 75-78, 2007.

2. Shi Z, Yuan B, Zhao D, Taylor AW, Lin J and Watts GF: Familial hypercholesterolemia in China: Prevalence and evidence of underdetection and undertreatment in a community population. Int J Cardiol 174: 834-836, 2014.

3. Cuchel M, Bruckert E, Ginsberg HN, Raal FJ, Santos RD, Hegele RA, Kuivenhoven JA, Nordestgaard BG, Descamps OS, Steinhagen-Thiessen E, et al: Homozygous familial hypercholesterolaemia: New insights and guidance for clinicians to improve detection and clinical management. A position paper from the Consensus Panel on Familial Hypercholesterolaemia of the European Atherosclerosis Society. Turk Kardiyol Dern Ars 43: 1-4, 2015 (In Turkish)

4. Page MM, Bell DA, Hooper AJ, Watts GF and Burnett JR: Lipoprotein apheresis and new therapies for severe familial hypercholesterolemia in adults and children. Best Pract Res Clin Endocrinol Metab 28: 387-403, 2014.

5. Vuorio A, Tikkanen MJ and Kovanen PT: Inhibition of hepatic microsomal triglyceride transfer protein-a novel therapeutic option for treatment of homozygous familial hypercholesterolemia. Vasc Health Risk Manag 10: 263-270, 2014.

6. Gidding SS: The complexities of homozygous familial hypercholesterolemia management. Pediatr Transplant 20: 1020-1021, 2016.

7. France M: Homozygous familial hypercholesterolaemia: Update on management. Paediatr Int Child Health 36: 243-247, 2016.

8. France M, Rees A, Datta D, Thompson G, Capps N, Ferns G, Ramaswami U, Seed M, Neely D, Cramb R, et al: HEART UK statement on the management of homozygous familial hypercholesterolaemia in the United Kingdom. Atherosclerosis 255 128-139, 2016.
9. Zhao X, Bu L, Qin S, Kong D, Fan B and Ge J: Early development of xanthoma and coronary disease in a young female with homozygous familial hypercholesterolemia. Int J Cardiol 176: e15-e19, 2014.

10. Jiang L, Gao F, Hu LB, Sun LY, Pan XD, Lin J, Zhang HB, Yong Q, Wang Q, Yang Y, et al: Seven-year clinical follow-up of a Chinese homozygous familial hypercholesterolemia child with premature xanthomas and coronary artery disease-a need for early diagnosis and aggressive treatment. Int J Cardiol 177: 188-191, 2014.

11. Vallejo-Vaz AJ, Kondapally Seshasai SR, Cole D, Hovingh GK, Kastelein JJ, Mata P, Raal FJ, Santos RD, Soran H, Watts GF, et al: Familial hypercholesterolaemia: A global call to arms. Atherosclerosis 243: 257-259, 2015.

12. Watts GF, Gidding S, Wierzbicki AS, Toth PP, Alonso R, Brown WV, Bruckert E, Defesche J, Lin KK, Livingston M, et al: Integrated guidance on the care of familial hypercholesterolaemia from the International FH Foundation. Eur J Prev Cardiol 22: 849-854, 2015.

13. Ito MK and Watts GF: Challenges in the diagnosis and treatment of homozygous familial hypercholesterolemia. Drugs 75: 1715-1724, 2015.

14. Besseling J, Sjouke B and Kastelein JJ: Screening and treatment of familial hypercholesterolemia-Lessons from the past and opportunities for the future (based on the Anitschkow Lecture 2014). Atherosclerosis 241: 597-606, 2015.

15. Cuchel M, Meagher EA, du Toit Theron H, Blom DJ, Marais AD, Hegele RA, Averna MR, Sirtori CR, Shah PK, Gaudet D, et al: Efficacy and safety of a microsomal triglyceride transfer protein inhibitor in patients with homozygous familial hypercholesterolaemia: A single-arm, open-label, phase 3 study. Lancet 381: 40-46, 2013.

16. Raal FJ, Honarpour N, Blom DJ, Hovingh GK, Xu F, Scott R, Wasserman SM and Stein EA; TESLA Investigators: Inhibition of PCSK9 with evolocumab in homozygous familial hypercholesterolaemia (TESLA Part B): A randomised, double-blind, placebo-controlled trial. Lancet 385: 341-350, 2015.

(i) This work is licensed under a Creative Common International (CC BY-NC-ND 4.0) License. 